\title{
Efectividad de hongos micorrizo-arbusculares nativos en suelos con diferentes usos agropecuarios
}

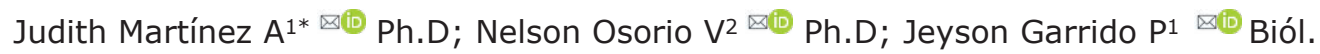

${ }^{1}$ Corporación Colombiana de Investigación Agropecuaria (Agrosavia), Grupo de investigación Raíces del Futuro, Cereté, Córdoba, Colombia.

2Universidad Nacional de Colombia, Medellin, Antioquia, Colombia

*Correspondence: jcmartinez@agrosavia.co

Recibido: Julio 2018; Aceptado: Marzo 2019; Publicado: Junio 2019.

\section{RESUMEN}

Objetivo. Determinar la efectividad de hongos micorrizo-arbusculares (HMA) de suelos con diferentes usos agropecuarios, en el valle medio del río Sinú, Colombia. Materiales y métodos. Bajo condiciones de invernadero se realizó el experimento, donde se utilizó como planta indicadora Leucaena leucocephala, la cual se sembró en materos con suelo de un Andisol (horizonte B) usado como sustrato. Este sustrato, se inoculó separadamente con muestras de siete suelos (horizonte Ap), como fuente de HMA, provenientes del Centro de Investigación Turipaná de Agrosavia. Como controles se incluyeron inoculación con HMA Glomus fasciculatum y uno no-inoculado El efecto de la inoculación micorrizal se evaluó mediante el monitoreo del contenido de P foliar en las plantas de L. leucocephala, en función del tiempo hasta 64 días cuando se determinó la masa seca de su parte aérea y la colonización micorrizal. Resultados. El crecimiento de $L$. leucocephala y el contenido de $P$ foliar fue significativamente superior cuando el suelo se inoculó con G. fasciculatum, en comparación a aquel observado con los demás tratamientos. De igual forma, la colonización micorrizal fue muy alta en las raíces de L. leucocephala que crecieron en el suelo inoculado con G. fasciculatum e inferior en los otros tratamientos. Conclusiones. Los resultados indican que los HMA nativos de suelos con diferentes usos, provenientes del C.I Turipaná de Agrosavia, exhibieron bajo potencial para desarrollar simbiosis micorrizal, lo que limitó el crecimiento y la concentración de $\mathrm{P}$ foliar en la planta hospedera.

Palabras clave: Fósforo, inoculación del suelo, Leucaena leucocephala, micorrizas arbusculares (Fuente: AGROVOC).

\begin{abstract}
Objective. To determine the effectiveness of mycorrhizal-arbuscular fungi (AMF) of soils with different agricultural uses, in the middle valley of the Sinú river, Colombia . Materials and methods. The experiment was carried out under greenhouse conditions, where Leucaena leucocephala was used as the indicator plant, which was planted in masons with an Andisol soil (horizon B) used as a substrate. This substrate was inoculated separately with samples from seven soils (Ap horizon), as a source of AMF, from the Turipaná Research Center in Agrosavia. As controls we included inoculation with HMA Glomus fasciculatum and one non-inoculated one. The effect of mycorrhizal inoculation was evaluated by monitoring the foliar P content in L. leucocephala plants, as a function of time to 64 days, when the dry mass of its aerial part and mycorrhizal colonization was determined. Results. The growth of L. leucocephala and the foliar P content was significantly higher when the soil was inoculated with G. fasciculatum, in comparison to that observed with the other treatments. Likewise, mycorrhizal colonization was very high in the roots of L. leucocephala that grew in the soil inoculated with G. fasciculatum and lower in the other treatments. Conclusions. The results indicate that the native AMF of soils with different uses, from Turipaná, exhibited low potential to develop mycorrhizal symbiosis, which limited the growth and concentration of leaf $\mathrm{P}$ in the host plant.
\end{abstract}

Keywords: Arbuscular mycorrhiza, Leucaena leucocephala, phosphorus, soil inoculation (Source: AGROVOC).

Como citar (Vancouver)

Martínez J, Osorio VN, Garrido PJ. Efectividad de hongos micorrizo-arbusculares nativos en suelos con diferentes usos agropecuarios. Rev MVZ Cordoba. 2019; 24(2):7256-7261. DOI: https://doi.org/10.21897/rmvz.1703

(C)EI (los) autor (es), Revista MVZ Córdoba 2019. Este artículo se distribuye bajo los términos de la licencia internacional Creative Commons Attribution 4.0 (https://creativecommons.org/licenses/by-sa/4.0/), que permite el uso sin restricciones, la distribución y la reproducción en cualquier medio, siempre que se otorgue el crédito apropiado al autor o autores originales y la fuente. 


\section{INTRODUCCIÓN}

La degradación de suelos afecta 3600 millones de hectáreas en el mundo, lo que constituye aproximadamente la cuarta parte de toda la tierra (1). Alrededor del 23\% de la tierra cultivable en el mundo ha sido afectado por degradación; en consecuencia, se han disminuido los niveles de productividad. Cerca del $16 \%$ de las tierras degradadas se encuentran en América Latina, lo cual representa el tercer lugar después de Asia y África (2). En Colombia, la actividad productiva agropecuaria ha estado acompañada de procesos de deterioro del entorno natural, que afectan su sostenibilidad ambiental y económica. Los departamentos de la región Caribe están entre los más erosionados del país, la mayoría presentan cifras de degradación entre 78 y $100 \%$, como consecuencia de circunstancias históricas, socioeconómicas y ambientales, tales como relieve, clima, viento, entre otras (3).

En América Latina, Colombia es el país con más alto porcentaje de suelo dedicado a las pasturas para la producción ganadera (4). En la región Caribe, este sistema de producción ocupa el $51 \%$ de su territorio (5) y juega un papel importante en la economía del país, ya que representa cerca del $35 \%$ del hato nacional, aporta el $40 \%$ del volumen de leche fresca y el $38 \%$ de carne. Sin embargo, a pesar de la importancia que representa este renglón productivo en la economía nacional, su sector primario enfrenta limitantes que influyen en la competitividad y sostenibilidad de los indicadores productivos, particularmente lo relacionado con procesos de degradación de suelos y praderas. Esto se refleja en una baja eficiencia biológica y económica de los sistemas ganaderos, debido a la baja calidad nutricional y oferta de forraje durante la época seca del año.

Dada la importancia de la ganadería en la economía de la región, se hace necesario la implementación de alternativas de manejo que permitan la conservación y/o recuperación de suelos. La sensibilidad de los indicadores biológicos del suelo permite observar los efectos de las prácticas de manejo en los sistemas, como una respuesta simultánea a cambios en las características físicas y químicas de este. Uno de estos indicadores es la formación de la asociación micorrizal entre hongos del suelo y sus plantas hospederas.

La importancia de los hongos micorrízicos arbusculares (HMA) en la nutrición de gramíneas y leguminosas forrajeras, especialmente en la toma de fósforo, amonio y otros elementos poco móviles en el suelo, ha sido objeto de muchos estudios (6). El aumento del volumen de suelo explorado, gracias al gran número de finas hifas de HMA, parece ser, en gran parte, responsable de las considerables diferencias en supervivencia, velocidad de crecimiento y rendimientos entre plantas micorrizadas y no-micorrizadas, particularmente en suelos degradados. La simbiosis efectiva entre gramíneas y HMA ha sido asociada con la recuperación de suelos degradados gracias a que la planta tiene una mejor nutrición por el acceso a un mayor volumen de suelo (7).

Por lo anterior, esta investigación buscó determinar la efectividad de HMA de suelos con diferentes usos (bosque, cultivos transitorios de maíz y algodón, pradera degradada y sistemas silvopastoriles de diferente complejidad estructural) del departamento de Córdoba.

\section{MATERIALES Y MÉTODOS}

Ubicación. Se estableció un experimento en el invernadero de la Universidad Nacional de Colombia, sede Medellín (6015 ' N, 75035' W y 1495 m de altitud). Muestras de suelo (horizonte $B$ ) de un Andisol se utilizaron para llenar materos a razón de $690 \mathrm{~g} /$ matero. El suelo fue secado al aire, tamizado a $4 \mathrm{~mm}$, mezclado en proporción 1:1 con cuarzo, y esterilizado dos veces en autoclave a $120^{\circ} \mathrm{C}$ y $0.1 \mathrm{MPa}$ durante una hora, con un periodo de 24 horas entre cada esterilización (8). El suelo tuvo un $\mathrm{pH}(1: 1$, agua) de 5.8 y se realizó una isoterma de adsorción de $\mathrm{P}$ (9) para determinar la cantidad de $P$ requerida para obtener una concentración de $P$ en la solución del suelo de $0.02 \mathrm{mg} \mathrm{L}^{-1}$; esta concentración se considera óptima para la actividad micorrizal (10). Al momento de la siembra, se aplicó $\mathrm{P}$ a razón de $588 \mathrm{mg}$ $\mathrm{kg}^{-1}$, como fuente de $\mathrm{P}$ se utilizó $\mathrm{KH} 2 \mathrm{PO}$. El sustrato presentó los siguientes resultados del análisis de suelos: materia orgánica 5.5\% (Walkley \& Black); P $22 \mathrm{mg} \mathrm{kg}^{-1}$ (Bray II); Ca, Mg y K 5.2, 0.9 y $0.31 \mathrm{cmolc} \mathrm{kg}^{-1}$ (Acetato de amonio), respectivamente.

El suelo de los materos fue inoculado separadamente con $30 \mathrm{~g} /$ matero de muestras de suelo superficial tomadas de los primeros $10 \mathrm{~cm}$ de profundidad (horizonte Ap) del Centro de Investigación Turipaná de Agrosavia ( $8^{\circ} 50^{\prime} \mathrm{N}$, $75^{\circ} 47^{\prime} \mathrm{W}$ y $15 \mathrm{~m}$ de altitud) ubicado en el municipio de Cereté (Córdoba, Colombia). Los suelos estaban sometidos a diferentes usos y manejo así: (i) pradera degradada (Pd) con Dichanthium aristatum bajo ganadería intensiva por más de 15 años, (ii) sistema intensivo de maíz-algodón (MA) en alternancia, (iii) bosque secundario (Bs) de 14 años, (iv) pradera ( $\mathrm{Pr}$ ) en rotación de pasto $D$. aristatum y Panicum maximum, (v) sistema silvopastoril constituido por pasturas de $D$. aristatum y $P$. maximum con especies arbóreas $(\mathrm{Pr}+\mathrm{A})$, (vi) sistema silvopastoril constituido por pasturas de $D$. aristatum y $P$. maximum con especies arbóreas y arbustivas $(\mathrm{Pr}+\mathrm{A}+\mathrm{a})$, (vii) sistema silvopastoril constituido por pasturas de $D$. aristatum y $P$. maximum con especies arbóreas, arbustivas y maderables $(\mathrm{Pr}+\mathrm{A}+\mathrm{a}+\mathrm{M})$. Como control positivo, se usó un inoculo crudo de Glomus fasciculatum (viii), de la colección del Laboratorio de Microbiología de la Universidad Nacional de Colombia, Sede Medellín; este inoculo contenía 40 propágulos infectivos (esporas, micelio extraradical y raíces colonizadas) por gramo, lo cual fue determinado por la técnica del número más probable (11). Adicionalmente, se incluyó como control negativo (ix) un tratamiento no-inoculado.

En cada matero se sembraron tres semillas germinadas de Leucaena leucocephala, las cuales fueron previamente escarificadas con ácido sulfúrico por 30 minutos y lavadas seis veces con agua destilada. Ésta se empleó como planta indicadora por su muy alta dependencia micorrizal y rápido crecimiento (10). Los materos recibieron agua para mantener el suelo entre $50-60 \%$ de la máxima capacidad de retención de agua. Una vez por semana se aplicaron $25 \mathrm{~cm}^{3}$ de solución Hoagland libre de $\mathrm{P}(10)$.

Efecto inoculación. Para evaluar el efecto de la inoculación micorrizal se monitoreó el contenido de $P$ foliar en el cuarto pínulo de la hoja joven más desarrollada de las plantas de L. leucocephala (10) en función del tiempo $(20,31,42,53$ y 64 días después de la siembradds-). A los 64 dds, se determinó la masa seca de la parte aérea de las plantas de $L$. leucocephala luego de secar en estufa $\left(60^{\circ} \mathrm{C}\right)$ durante 72 horas. 
Colonización. La colonización micorrizal de las raíces de L. leucocephala, fue determinada luego de clarificarlas con $\mathrm{KOH}(10 \%)$ (12) y teñirlas con fucsina ácida (13) y luego determinar la extensión de la colonización mediante el método del intercepto de la cuadrícula (14).

Diseño experimental. Se utilizó un diseño experimental completamente al azar, los tratamientos consistieron en las inoculaciones separadas con siete muestras de los suelos descritos anteriormente, la inoculación con $G$. fasciculatum y el control no-inoculado, es decir nueve tratamientos. Cada tratamiento tuvo cuatro repeticiones, para un total de 36 unidades experimentales.

Los datos fueron sometidos a análisis de varianza y la comparación de medias se realizó con la prueba de mínima diferencia significativa (LSD), con un nivel de significancia $(P)$ de 0.05 . Los análisis se realizaron con el paquete estadístico SAS versión 8 .

\section{RESULTADOS}

El contenido de $\mathrm{P}$ foliar en plantas de $L$. leucocephala fue significativamente superior cuando el suelo se inoculó con G. fasciculatum en comparación a aquel observado con los demás tratamientos (Figura 1). Los efectos favorables de la inoculación con este hongo micorrizal fueron detectados a partir del día 42 dds; este efecto se mantuvo hasta el final del periodo de evaluación (64 dds) (Tabla 1).

Tabla 1. Niveles de significancia de los análisis de varianza y coeficientes de variación (CV) para las variables bajo estudio.

\begin{tabular}{cccccccc}
\hline Variable & P20 & P31 & P42 & P53 & P64 & MSA & CM \\
\hline$p$-value & NS & NS & 0.01 & 0.0001 & $<0.0001$ & $<0.0001$ & $<0.0001$ \\
CV (\%) & 22.2 & 20.8 & 27.1 & 14.2 & 20.1 & 19.3 & 22.4 \\
LSD & 0.76 & 0.69 & 0.89 & 0.39 & 0.56 & 0.18 & 2.61 \\
\hline
\end{tabular}

P20-64: contenido de P foliar en los días de muestreo; MSA: masa seca aérea; CM: colonización micorrizal.

En las dos primeras fechas de muestreo ( 20 y $31 \mathrm{dds}$ ), el contenido de $\mathrm{P}$ foliar no fue influenciado significativamente por la fuente de inóculo utilizada. La inoculación con las muestras de suelos no incrementó significativamente el contenido de $\mathrm{P}$ foliar con respecto al control no-inoculado en las diferentes fechas de muestreo (Figura 1).

El efecto de las fuentes de inóculo usadas también fue fácilmente observado en los registros de masa seca aérea de $L$. leucocephala (Figura 2 ) y corroboran los resultados anteriormente señalados. Las plantas de L. leucocephala que crecieron en suelo inoculado con $G$. fasciculatum exhibieron una masa seca aérea significativamente superior a la de los otros tratamientos (Tabla 1). Al inocular el suelo con las otras fuentes de inóculo no se incrementó la masa seca aérea por encima de la detectada con el tratamiento control (no-inoculado).

La colonización de raíces de $L$. leucocephala por HMA fue significativamente diferente en función a las fuentes de inóculo (Tabla 1, Figura 3). La colonización micorrizal fue muy alta en las raíces de $L$. leucocephala que crecieron en el suelo inoculado con G. fasciculatum (63\%). En las raíces de plantas del control no-inoculado no se detectó colonización micorrizal. La colonización micorrizal de las raíces con los otros tratamientos fuentes de inóculo fue muy baja o nula $(\leq 4 \%)$.

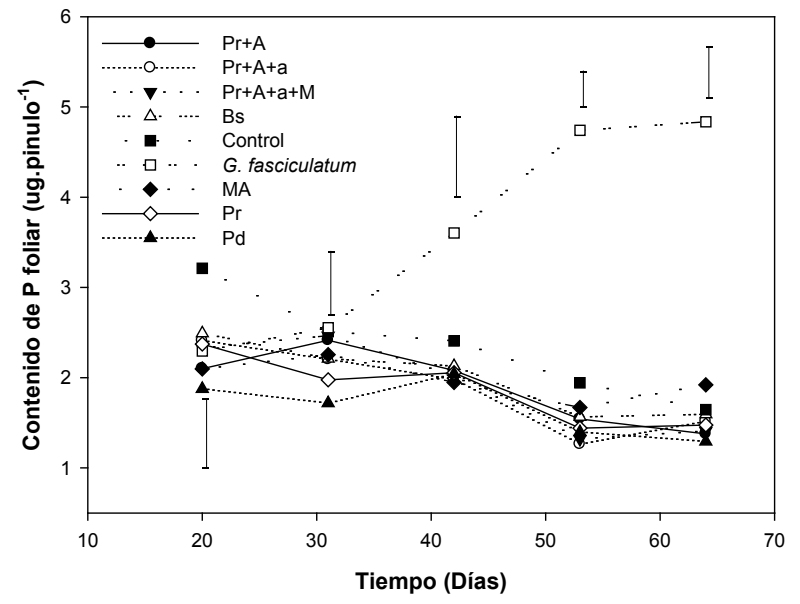

Figura 1. Contenido de $P$ foliar en pínulos de leucaena en función de la inoculación con muestras de suelos bajo diferentes uso y manejo provenientes del valle medio del Sinú a través del tiempo de muestreo. Los datos de las dos primeras fechas no presentaron diferencias en función de los tratamientos. Las barras representan la diferencia mínima significativa (LSD) para $p=0.05$ entre medias.

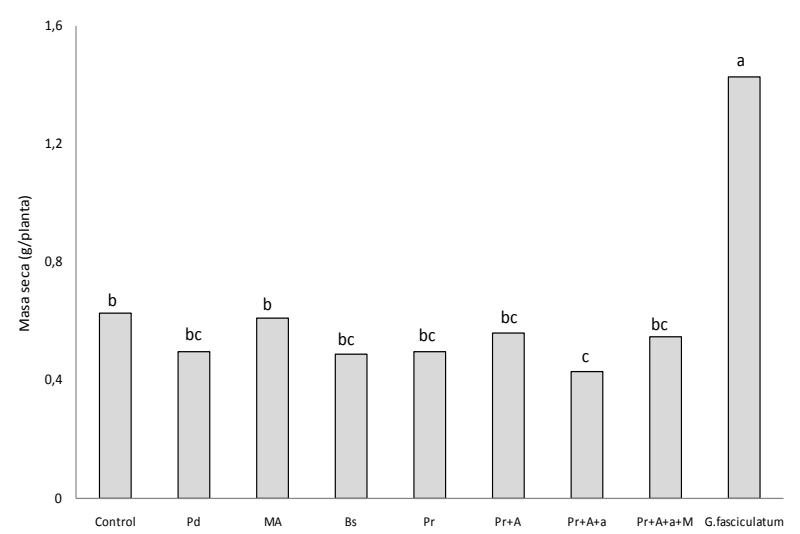

Figura 2. Masa seca aérea de leucaena en función de la inoculación con muestras de suelos bajo diferentes uso y manejo provenientes del valle medio del Sinú.

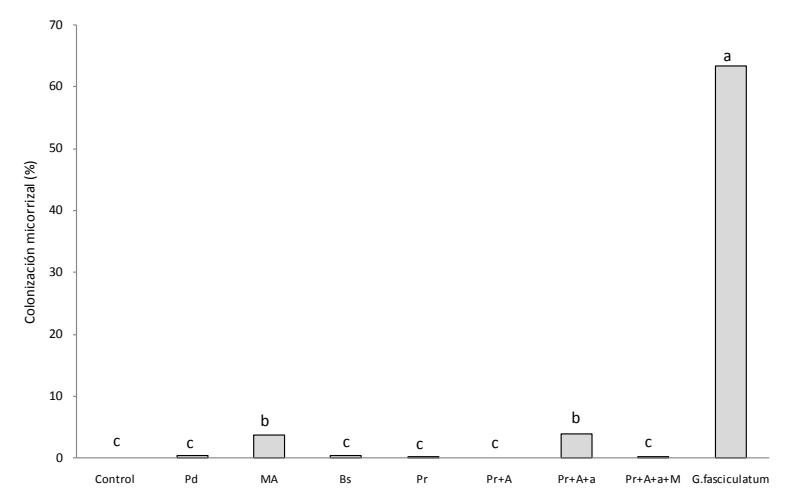

Figura 3. Colonización micorrizal de raíces de leucaena en función de la inoculación con muestras de suelos bajo diferentes uso y manejo provenientes del valle medio del Sinú. 


\section{DISCUSIÓN}

Los resultados no soportan la hipótesis de que la efectividad de HMA de los suelos está en función del tipo de cobertura y manejo del suelo y que puede cambiar de acuerdo al grado de complejidad de los sistemas donde los hongos están presentes. Los suelos usados como fuente de inóculo micorrizal exhibieron una baja efectividad micorrizal y no superaron al control noinoculado. Esta baja efectividad micorrizal se puede deber a las condiciones físico-químicas de los suelos estudiados. Su fracción fina está dominada por arcillas del tipo $2: 1$, las cuales exhiben una alta capacidad de expansión y contracción según sea el nivel de humedad del suelo, y dada la marcada estacionalidad del periodo de lluvias y sequía esto se constituye en un factor importante en la dinámica física de estos suelos $(15,16,17,18)$.

Durante la época lluviosa cuando la arcilla está expandida se puede limitar la circulación de oxígeno en el suelo, lo cual afecta los HMA que son aeróbicos. Por otra parte, durante la época seca la contracción del material podría generar estrangulamiento de raíces de las plantas y eventualmente ruptura de las hifas de los hongos micorrizales. Varios autores $(19,20,21,22,23)$ han reportado que al disturbar mecánicamente el suelo las hifas de HMA se rompen y, en consecuencia, se reduce su viabilidad e infectividad. De igual manera, los suelos que fueron fuente de inóculo micorrizal están expuestos en mayor o menor grado a inundaciones periódicas y pueden permanecer saturados hasta por tres meses consecutivos al año. Tales condiciones anaeróbicas restringen el acceso de estos hongos al oxígeno, lo cual obviamente es desfavorable para su desarrollo (16).

Pruebas posteriores permitieron determinar que el número de esporas de HMA fue muy bajo en las muestras de suelo (11 a 22 esporas/g) usadas como fuente de inóculo micorrizal (Tabla 2), valores inferiores a los reportados por en estudios realizados en praderas de Bothriochloa pertusa (L) A. Camus de fincas ganaderas del municipio de Corozal en el departamento de Sucre, con registros de 15-60 esporas/g (24). Se puede inferir que las esporas presentes en los suelos usados como fuente de inóculo micorrizal hayan perdido su viabilidad y no sean capaces de formar la asociación micorrizal. Los valores de colonización encontrados en este estudio fueron menores del 5\%, esto explicaría la baja efectividad micorrizal detectada en esos suelos; estos resultados son contrastantes con los observados en praderas de $D$. aristatum en fincas de la zona costanera del departamento de Sucre, donde la colonización exhibió valores superiores al $20 \%$ (25), lo cual es atribuible a las diferencias entre ambientes y las características físicoquímicas de los suelos.

Los suelos evaluados, antes del establecimiento de los sistemas actuales de producción estuvieron bajo ganadería intensiva por no menos de 15 años (26). Dada las condiciones de deficiente drenaje interno y externo estos suelos fueron compactados (27). Se considera que en su momento las plantas de los sistemas silvopastoriles se establecieron porque el suelo químicamente es muy fértil y esto les permitió tolerar esas condiciones físico-químicas y atmosféricas en algunos periodos desfavorables (26). Las plantas de $L$. leucocephala tiene una muy alta dependencia de la asociación micorrizal para absorber P (10), esto es consistente con los resultados del presente estudio ya que al no haber suficiente inóculo micorrizal viable las plantas que dependen de los HMA pueden desaparecer del sistema, tal como ha ocurrido con L. leucocephala dentro de los sistemas silvopastoriles evaluados en este estudio.
Adicionalmente, el análisis de la concentración de $\mathrm{P}$ soluble en el suelo $\left(\mathrm{CaCl}_{2} 0.01 \mathrm{M}\right)$ permitió detectar que la disponibilidad de $\mathrm{P}$ en estos suelos es relativamente alta (Tabla 2 ) y está por encima del nivel óptimo $(0.02 \mathrm{mg}$ $\mathrm{L}^{-1}$ ) para la actividad micorrizal. Altas concentraciones de $P$ en la solución del suelo pueden disminuir la actividad y colonización de HMA en los suelos $(10,28)$.

Tabla 2. Número de esporas de HMA y concentración de P en la solución del suelo de muestras usadas como fuente de inóculo.

\begin{tabular}{|c|c|c|}
\hline Suelo/Cobertura & $\begin{array}{c}\text { Esporas HMA* } \\
\left(\# \mathbf{g}^{-1}\right)\end{array}$ & $\begin{array}{l}\text { Concentración de } \mathrm{P} \text { en la } \\
\text { solución** }\left(\mathrm{mg} \mathrm{L}^{-1}\right)\end{array}$ \\
\hline $\mathrm{Pr}+\mathrm{A}$ & 11 & 0.17 \\
\hline $\mathrm{Pr}+\mathrm{A}+\mathrm{a}$ & 12 & 0.19 \\
\hline $\mathrm{Pr}+\mathrm{A}+\mathrm{a}+\mathrm{M}$ & 14 & 0.23 \\
\hline Bs & 17 & 0.15 \\
\hline MA & 13 & 0.11 \\
\hline $\operatorname{Pr}$ & 20 & 0.35 \\
\hline $\mathrm{Pd}$ & 22 & 0.13 \\
\hline G. fasciculatum & 25 & 0.02 \\
\hline Control & 0 & 0.02 \\
\hline
\end{tabular}

* Método del tamizaje en húmedo

** $0.01 \mathrm{M} \mathrm{CaCl}_{2}$ y determinación por fosfomolibdato

El potencial simbiótico micorrizal de un suelo es el resultado del tipo de suelo, de las prácticas de uso y manejo del suelo (prácticas agrícolas, minería), condiciones fisco-químicas, ambientales, microbiológicas y por la presencia de especies vegetales que pueden favorecer o desfavorecer la asociación micorrizal $(29,30,31,32)$. Los anteriores aspectos, permiten explicar el porqué de la baja efectividad simbiótica micorrizal de las poblaciones nativas en los suelos estudiados. Estos resultados son comparables a los obtenidos (33) al inocular un sustrato de crecimiento con muestras de suelos degradados (como fuente de inóculo) que exhibían un bajo número de propágulos micorrizales infectivos y no hubo una buena colonización micorrizal y, por ende, no encontraron un efecto benéfico sobre la absorción de $P$ y el crecimiento vegetal de plantas de $L$. leucocephala.

En conclusión, los resultados indican que los HMA nativos de suelos con diferentes usos evaluados, exhibieron bajo potencial para desarrollar simbiosis micorrizal, lo que limitó el crecimiento y la concentración de $\mathrm{P}$ foliar en la planta hospedera.

\section{Conflicto de interés}

Los autores declaran no tener conflictos de interés

\section{Agradecimientos}

Este trabajo fue realizado con el apoyo financiero del Ministerio de Agricultura y Desarrollo Rural dentro del proyecto 200705209-866/1466, liderado por la Corporación Colombiana de investigación-Agrosavia. Las evaluaciones se realizaron en las instalaciones del Invernadero y del Laboratorio de Microbiología del Suelo de la Universidad Nacional de Colombia, sede Medellín. 


\section{REFERENCIAS}

1. Bai ZG, Dent DL, Olsson L, Schaepman ME. Proxy global assessment of land degradation. Soil Use and Management. 2008; 24(3):223-34. https:// doi.org/10.1111/j.1475-2743.2008.00169.x

2. MAD-IDEAM. Síntesis nacional de la degradación de los suelos por erosión en Colombia. [en línea]. 2015 [Acceso 12 de junio de 2018] URL disponible en: http://documentacion.ideam.gov.co/openbiblio/ bvirtual/023648/Sintesis.pdf

3. Ministerio de Ambiente, Vivienda y Desarrollo Territorial- MAVDT. Plan de Acción Nacional de Lucha Contra la Desertificación y la Sequía en Colombia. [en línea]. 2005 [Acceso 18 de julio de 2018] URL disponible en: http://www.minambiente.gov.co/images/ BosquesBiodiversidadyServiciosEcosistemicos/ pdf/Zonas-Secas/5596 250510 plan lucha desertificacion.pdf

4. FAOSTAT (Statistical Databases of the Food and Agriculture Organization of the United Nations). [en línea]. 2018. [Acceso 12 de junio de 2018] URL disponible en: http://www.fao.org/faostat/ en/\# country/44

5. IGAC. Los pastos es la cobertura que reina en los suelos de la región Caribe. Instituto Geográfico Agustín Codazzi [en línea] 2014 [Acceso 18 de julio de 2018]; URL disponible en: https://noticias.igac. gov.co/es/contenido/los-pastos-es-la-coberturaque-reina-en-los-suelos-de-la-region-caribe

6. Smith SE, Read DJ. Mycorrhizal Symbiosis [Internet]. Elsevier; 2002. URL Disponible en: http://dx.doi.org/10.1016/b978-0126528404/50001-2

7. Habte M, Fox RL, Huang RS. Determining vesicular-arbuscular mycorrhizal effectiveness by monitoring $\mathrm{P}$ status of subleaflets of an indicator plant. Communications in Soil Science and Plant Analysis. 1987; 18:1403-1420. DOI: https://doi. org/10.1080/00103628709367907

8. Montoya B, Osorio NW. Mycorrhizal dependency of avocado at different levels of soil solution phosphorus. Suelos Ecuatoriales. 2009; 39(1):43-147. https:// sites.google.com/site/suelosecuatoriales/descargade-articulos/volumen-39-1

9. Fox RL, Kamprath EJ. Phosphate Sorption Isotherms for Evaluating the Phosphate Requirements of Soils 1. Soil Sci Soc Am J. 1970; 34(6):902-907. DOI: https://doi.org/10.2136/sssaj1970.03615995003 400060025x

10. Osorio NW, Habte M. Synergistic effect of a phosphate-solubilizing fungus and an arbuscular mycorrhizal fungus on leucaena seedlings in an Oxisol fertilized with rock phosphate. Botany. 2013; 91(4):274-281. DOI: https://doi.org/10.1139/cjb$\underline{2012-0226}$
11. Ziane H, Meddad-Hamza A, Beddiar A, Gianinazzi S. Effects of Arbuscular Mycorrhizal Fungi and Fertilization Levels on Industrial Tomato Growth and Production. International Journal of Agriculture and Biology. 2017; 19(02):341-7. DOI: https://doi. org/10.17957/IJAB/15.0287

12. Phillips JM, Hayman DS. Improved procedures for clearing roots and staining parasitic and vesiculararbuscular mycorrhizal fungi for rapid assessment of infection. Transactions of the British Mycological Society. 1970; 55(1):158-161. DOI: https://doi. org/10.1016/S0007-1536(70)80110-3

13. Kormanik PP, Bryan WC, Schultz RC. Procedures and equipment for staining large numbers of plant root samples for endomycorrhizal assay. Can J Microbiol. 1980; 26(4):536-538. DOI: https://doi. org/10.1139/m80-090 PMid: 6155191

14. Giovannetti M, Mosse B. An evaluation of techniques for measuring vesicular arbuscular mycorrhizal infection in roots. New Phytologist. 1980; 84(3):489-500. DOI: http://dx.doi. org/10.1111/j.1469-8137.1980.tb04556.x

15. Martinez J. Efecto de tres sistemas de labranza sobre algunas propiedades físicas en un endoaquept, el desarrollo de las raíces y los rendimientos de maíz (Zea mays I.) y algodón (Gossypium hirsutum), en el valle medio del Sinú - Colombia. [Tesis de Maestría]. Universidad Nacional de Colombia; Palmira, Colombia: 2004.

16. Bola-os BM, Rivillas OC, Suárez VS. Identificación de micorrizas arbusculares en suelos de la zona cafetera Colombiana. Cenicafé 2000; 51(4):245262. https://www.cenicafe.org/es/publications/ arc051\%2804\%29245-262.pdf

17. Barea JM, Jeffries P. Arbuscular mycorrhizas in sustainable soil-plant systems. En: Varma A, HOCK $B$, ed. Mycorrhiza, structure, function, molecular biology and biotechnology. New York: Wiley, 1995. DOI: https://doi.org/10.1007/978-3-662-08897$\underline{523}$

18. Cajas Giron YS, Sinclair FL. Characerization of multistrata silvopasttoral systems on seasonally dry pastures in the Caribbean Region of Colombia. Agroforestry Systems 2001; 53:215-225. DOI: https://doi.org/10.1023/A:1013384706085

19. Alguacil MM, Lumini E, Roldan A, Salinas Garcia JR, Bonfante P, Bianciotto V. The impact of tillage practices on arbuscular mycorrhizal fungal diversity in subtropical crops. Ecol Appl. 2008; 18(2):527536. DOI: https://doi.org/10.1890/07-0521.1 PMid: 18488613 
20. Cobb AB, Wilson GW, Goad CL, Bean SR, Kaufman RC, Herald TJ, et al. The role of arbuscular mycorrhizal fungi in grain production and nutrition of sorghum genotypes: Enhancing sustainability through plant-microbial partnership. Agric Ecosyst Environ. 2016; 233:432-440. DOI: https://doi. org/10.1016/j.agee.2016.09.024

21. Osorio NW, Habte M. Effect of a phosphatesolubilizing fungus and an arbuscular mycorrhizal fungus on leucaena seedlings in tropical soils with contrasting phosphate sorption capacity. Plant Soil. 2015; 389(1-2):375-385. DOI: https://doi. org/10.1007/s11104-014-2357-5

22. Curaqueo G, Barea JM, Acevedo E, Rubio R, Cornejo P, Borie F. Effects of different tillage system on arbuscular mycorrhizal fungal propagules and physical properties in a Mediterranean agroecosystem in central Chile. Soil Tillage Res. $2011 ; 113(1): 11-18$. DOI: https://doi. org/10.1016/j.still.2011.02.004

23. Curaqueo G, Acevedo E, Cornejo P, Seguel A, Rubio R, Borie F. Tillage effect on soil organic matter, mycorrhizal hyphae and aggregates in a mediterranean agroecosystem. Cienc Suelo. 2010; 10(1):12-21. DOI: https://doi.org/10.4067/S0718$\underline{27912010000100002}$

24. Pérez CA, Botero LC, Cepero GM. Diversidad de micorrizas arbusculares en pasto colosuana (Bothriochloa pertusa)(L) A. Camus de fincas ganaderas del municipio de Corozal-Sucre. Rev MVZ Córdoba. 2012; 17(2):3024-3032. DOI: https:// doi.org/10.21897/rmvz.237

25. Pérez A, Peroza V. Micorrizas arbusculares asociadas al pasto angleton (Dichathium aristatum Benth) en fincas ganaderas del municipio de Tolú, SucreColombia. Rev MVZ Córdoba. 2013; 18(1):33623369. DOI: https://doi.org/10.21897/rmvz.199

26. Martínez J, Cajas YS, León JD, Osorio NW. Silvopastoral Systems Enhance Soil Quality in Grasslands of Colombia. Applied and Environmental Soil Science 2014; Article ID 359736. DOI: http:// dx.doi.org/10.1155/2014/359736
27. Cuadrado HR, Torregrosa L, Jiménez NC. Comparación bajo pastoreo con bovinos machos de ceba de cuatro especies de gramíneas del género Brachiaria. Rev MVZ Córdoba. 2004; 9(2):438-444. DOI: https://doi.org/10.21897/rmvz.485

28. Osorio Vega NW, Serna Gómez SL, Montoya Restrepo BE. Use of soil microorganisms as a biotechnological strategy to enhance avocado (persea americana)plant phosphate uptake and growth. Rev Fac Nac Agron. 2012; 65(2):6645-6657. http://www. scielo.org.co/scielo.php?script $=$ sci arttext\&pid $=$ S0304-28472012000200009

29. Pizano C, Mangan SA, Graham JH, Kitajima K. Host-specific effects of soil microbial filtrates prevail over those of arbuscular mycorrhizae in a fragmented landscape. Ecological Applications. Wiley; 2017; 27(6):1946-1957. DOI: http://dx.doi. org/10.1002/eap.1579

30. Svenningsen NB, Watts Williams SJ, Joner EJ, Battini F, Efthymiou A, Cruz Paredes C. Suppression of the activity of arbuscular mycorrhizal fungi by the soil microbiota. ISME J. 2018; 12(5):1296. DOI: https://doi.org/10.1038/s41396-018-0059-3 PMid: 29382946

31. Berdugo Silva EB. El uso de hongos micorrízicos arbusculares como una alternativa para la agricultura. Rev Bio Agro. 2009; 7(1):123-132. http://revistabiotecnologia.unicauca.edu.co/ revista/index.php/biotecnologia/article/view/110

32. Pérez CA, Vertel MM. Evaluación de la colonización de micorrizas arbusculares en pasto Bothriochloa pertusa (L) A. Camus. Rev MVZ Córdoba 2010; 15(3):2165-2174. DOI: https://doi.org/10.21897/ rmvz.303

33. Jaramillo P, Silva JM, Osorio NW. Potencial simbiótico y efectividad de hongos micorrizo arbusculares de tres suelos sometidos a diferentes usos. Rev Fac Nac Agron. 2004; 57(1):2203-2214. https:// revistas.unal.edu.co/index.php/refame/article/ view/24175 